\title{
Mechanism of MCP-1 in Acute Lung Injury and Advanced Therapy by Drug-Loaded Dextrin Nanoparticle
}

\author{
Zheng Cao, ${ }^{1}$ Jing-Lan Liu, ${ }^{2}$ Shen $W u^{1}{ }^{1}$ and Qiao Wang $\mathbb{D}^{1}$ \\ ${ }^{1}$ Emergency Department, Shanghai Ninth People's Hospital, School of Medicine, Shanghai Jiao Tong University, Shanghai, China \\ ${ }^{2}$ The International Peace Maternity \& Child Health Hospital of the China Welfare Institute, Shanghai, China
}

Correspondence should be addressed to Qiao Wang; wangqiao1298@126.com

Received 11 July 2018; Revised 7 August 2018; Accepted 15 August 2018; Published 27 September 2018

Academic Editor: Jianxun Ding

Copyright (c) 2018 Zheng Cao et al. This is an open access article distributed under the Creative Commons Attribution License, which permits unrestricted use, distribution, and reproduction in any medium, provided the original work is properly cited.

\begin{abstract}
Objective. To observe the expression of monocyte chemotactic protein 1 (MCP-1) in acute lung injury (ALI) rat model, to characterize its effect on the development and progression of ALI, and to identify the potential new drug delivery approach during in vivo experiment. Method. The effects of different doses of lipopolysaccharide (LPS) on human pulmonary artery endothelial cells (HPAEC) were tested. For the animal experiments, thirty Sprague-Dawley (SD) rats were divided into physiological saline control group (NC group), the LPS model group (L group), the antagonist RS102895 combined with LPS group ( $\mathrm{R}+\mathrm{L}$ group), and the antagonist RS102895-loaded polyaldehyde dextran nanoparticles combined with LPS group (DNPR + L group). The blood gas analysis and dry/wet weight ratio were detected 24 hours after interventions. The levels of inflammatory factors, tumor necrosis factor- $\alpha$ (TNF- $\alpha$ ) and interleukin- $1 \beta$ (IL- $1 \beta$ ), were tested by ELISA. The expression of monocyte chemoattractant protein-1 (MCP-1) in lung tissues was examined through Western blot, and the change of MCP-1 mRNA expression level was detected by performing RT-PCR. Result. LPS was responsible for inducing ALI in rats, and the degree of cell damage was dose-dependent. Blood gas analysis of $\mathrm{L}$ group showed that $\mathrm{PaO} 2$ and $\mathrm{PaO} 2 / \mathrm{FiO} 2$ levels were significantly lower than those of the NC group $(P<0.05)$, while the dry/wet weight ratio of lung tissues in L group increased $(P<0.05)$. Inflammatory factors including TNF- $\alpha$ and IL-1 $\beta$ and the expression of MCP- 1 in both protein and mRNA levels were higher in $\mathrm{L}$ group than in the NC group $(P<0.05)$. The inhibition of the interaction between MCP-1 and chemokines receptor 2 (CCR2) by antagonist RS102895 can significantly alleviate the ALI in rats, which is accompanied by a significant decrease of inflammatory factors and MCP-1 expression $(P<0.05)$. Compared with $\mathrm{R}+\mathrm{L}$ group, treatment with DNPR and LPS combination significantly improved the condition of rats and decreased the level of TNF- $\alpha$, IL- $1 \beta$, and MCP-1 expression $(P<0.05)$. Conclusion. In ALI, RS102895 can inhibit the MCP-1/CCR2 interaction, therefore, retarding the progress of ALI. Because of the high transfection efficiency of inhibitor RS102895packgaed by polyaldehyde dextran nanoparticles, this phenomenon particularly reached a significant level. The results imply new insights for the treatment of ALI.
\end{abstract}

\section{Introduction}

Acute lung injury (ALI) and its severe stage, acute respiratory distress syndrome (ARDS), are defined as acute pneumonia and tissue damaged disease. The clinical symptoms include acute hypoxemic respiratory failure, reduced pulmonary compliance, excessive pulmonary inflammation, pulmonary edema, and diffuse alveolar damage due to an imbalance of pulmonary gas exchange and blood flow $[1,2]$. ALI is mainly caused by acute inflammations induced by infection, trauma, or gastric acid sucking [3-5]. Up until now, researchers have made great progress in the pathogenesis of ALI and ARDS by proposing various treatments; however, the morbidity and mortality of these diseases remain high [6-8].

The pathogenesis of ALI is very complicated as alveolar macrophages play a key role in the development of ALI. Alveolar macrophage is the most common nonparenchymal cells in lung tissue. Once activated by bacterial or viral infection, macrophages generate and release a large number of inflammatory cytokines and chemokines, at the same time, transport a large number of leukocytes to the lesion $[9,10]$. Meanwhile, cell signaling is activated through multilevel 
signal transduction with a cascade of inflammatory transcription factors activation, inflammatory cytokines genes overexpression, excessive immune response, and inflammatory response [11]. Chemokines are crucial in leukocyte recruitment, activation, and related functions as well as in the progression of inflammation. Previous work showed dextran sulfate-graft-methotrexate conjugate therapeutic value and targeted the SR-expressed activated macrophages in the treatment of collagen-induced arthritis [12]. On account of the small size, nanoparticles possess greater physical activity, enabling contact between the molecules and target cells, and improving therapeutic efficacy $[13,14]$. Self-assembled nanoparticles with chemokine receptor antagonist RS102895 and polyaldehyde dextran were prepared to improve lung injury for this study.

In this study, in vitro HPAEC model is used to study the effect of LPS on cell damage. Then a rat model of LPS-induced ALI was built and evaluated by blood gas analysis. Furthermore, we compared the inhibition efficiency between direct administration and polyaldehyde dextrancoated nanoparticles with antagonist RS102895, expecting to find a better approach to achieve high-efficiency inhibitor delivery with less extra injury. Finally, in this study, we discuss the underlying mechanism of MCP-1 in ALI rat model and provide new therapeutic ideas for the clinical treatment of ALI.

\section{Materials and Methods}

Cell lines. The culture medium of HPAEC and endothelial cells were purchased from ScienCell (Carlsbad, CA, USA).

(1) Animals. Thirty adult male Sprague-Dawley rats weighing 200-220 g were purchased from Shanghai SLAC Laboratory Animal Co., Ltd (Shanghai, China).

(2) Reagents. DPBS and trypsin were purchased from Gibco (Carlsbad, CA, USA); LPS was purchased from Sigma (St. Louis, MO, USA); CCK-8 ELISA kit was purchased from Beyotime Biotechnology (Shanghai, China); Annexin VAPC/7-AAD kit was purchased from BioLegend (San Diego, CA, USA); TNF- $\alpha$ and IL- $1 \beta$ ELISA kit were purchased from R\&D system (Minneapolis MN, USA); MCP-1 antibody was purchased from BioLegend (San Diego, CA, USA); the design and synthesis of MCP-1 mRNA primer were by Invitrogen (Carlsbad, CA, USA); RNA and RNAase H-reverse transcriptase were purchased from Invitrogen (Carlsbad, CA, USA); and RS102895 was purchased from Santa Cruz Biotechnology, Inc. (Santa Cruz, CA, USA). Dextran was purchased from Nobilus, Kutno Company. Sodium periodate was purchased from Sigma-Aldrich Company. Ethylene glycol was purchased from Chempur, Piekary Slaskie Company.

2.1. Cell Treatment. Logarithmic phase HPAEC cells in good conditions were digested and adjusted to a density of $1 \times 10^{5}$ cells per $\mathrm{mL}$ with culture media supplemented with $10 \%$ fetal bovine serum and $1 \%$ penicillin and streptomycin and then were plated $1 \mathrm{~mL}$ per well into a 12-well plate. The cells were grown until confluence reached $70 \%-80 \%$ before the LPS treatment. The supernatant of culture media was removed, and the cells were washed once using Dulbecco's phosphate-buffered saline (DPBS). Then cells were divided into control group and experimental groups: experimental groups were treated with different concentrations of LPS solutions $(100 \mathrm{ng} / \mathrm{mL}$, $500 \mathrm{ng} / \mathrm{mL}$, and $1 \mu \mathrm{g} / \mathrm{mL}$ ), while the control group was treated with culture media. Each treatment was done in triplicate. All groups of cells were cultured in a humidified atmosphere with $5 \% \mathrm{CO}_{2}$ at $37^{\circ} \mathrm{C}$.

2.2. Cell Viability. After LPS interventions for $24 \mathrm{~h}$, the supernatant was removed, and the cells were washed twice using DPBS. $1.1 \mathrm{~mL}$ CCK-8 working solutions $(0.1 \mathrm{~mL}$ CCK : $1 \mathrm{~mL}$ culture medium) were then added to each well. Culture supernatants were collected 4 hours after incubation and added into a 96-well plate (100 $\mu \mathrm{L}$ per well). Absorbance for collected culture supernatants was measured at $450 \mathrm{~nm}$.

2.3. Apoptosis Assay. The cells were harvested $24 \mathrm{~h}$ after LPS treatments, washed twice with binding buffer, and suspended in $500 \mu \mathrm{L}$ annexin $\mathrm{V}$ binding buffer. Following that, the cells were stained with $2 \mu \mathrm{L}$ Annexin V-APC and $5 \mu \mathrm{L}$ $7-\mathrm{AAD}$ at room temperature for $10 \mathrm{~min}$ protected from light. The stained cells were then subjected to flow cytometry for analysis.

2.4. ALI Model of SD Rats. Thirty SD rats were randomly divided into four groups: (1) normal saline control group (NC group) which rats received intraperitoneal injection of $2 \mathrm{~mL} / \mathrm{kg}$ saline; (2) LPS group which rats received $10 \mathrm{mg} / \mathrm{kg}$ LPS by intraperitoneal injection; (3) antagonist RS102895 combined with LPS ( $\mathrm{R}+\mathrm{L}$ group) which rats received intraperitoneal injection of $2 \mathrm{~mL} / \mathrm{kg}$ RS102895 and $10 \mathrm{mg} / \mathrm{kg}$ LPS injection 24 hours after the antagonist treatment; and (4) the antagonist RS102895-loaded polyaldehyde dextran nanoparticles combined with LPS (DNPR $+\mathrm{L}$ group) which rats received intraperitoneal injection with $2 \mathrm{mg} / \mathrm{kg}$ RS102895 formulated nanoparticle and LPS $(10 \mathrm{mg} / \mathrm{kg})$ injection 24 hours after nanoparticle injection. Rats were sacrificed 24 hours after each intervention, and samples were collected for further use.

2.5. Preparation of RS102895-Loaded NPs. Sodium periodate $(2 \mathrm{~g})$ was added to $200 \mathrm{~mL}$ of dextran $(50 \mathrm{mg} / \mathrm{mL}$ dissolved in distilled water). After 1 hour, ethanediol was added to the mixture. Secure the mixture with the dialysis membrane bag and precipitate the distilled water for 3 days and dried for 24 hours after participation. The characteristics of polyaldehyde dextran (PAD) were tested by hydroxylamine hydrochloride method. Dried polyaldehyde dextran (1 g) was dissolved in distilled water $\left(10 \mathrm{~mL}, 30^{\circ} \mathrm{C}\right)$ and mixed with $0.01 \mathrm{~g} / \mathrm{mL}$ RS102895 solution. After stirred for $30 \mathrm{~min}, \mathrm{pH}$ was adjusted to 7.4 with sodium hydroxide or hydrochloric acid. The mixture was set for dialysis for $30 \mathrm{~min}$.

2.6. Measurement of Characteristics of RS102895 Formulations. The drug encapsulation efficiency (EE) and loading efficiency (LE) were calculated to characterize the efficiency 
of NPs production. LE was defined as the ratio of the mass of RS102895 in NPs to the total mass of NPs, and EE was the percentage of the mass of RS102895 to the mass of the total used RS102895 for NPs preparation. The mass and purity of RS102895 in NPs were evaluated by HPLC (Malvern Instruments, UK). All measurements were performed in triplicate.

2.7. Arterial Blood Gas Analysis. 24 hours after LPS treatments, $0.5 \mathrm{~mL}$ blood was drawn from rat celiac artery by $1 \mathrm{~mL}$ syringe and used for blood gas analysis.

2.8. Lung Wet/Dry Weight Ratio. After scarifying the rats, the inferior lobe of the right lungs was excised, cleaned, and weighed to obtain wet weight $(\mathrm{W})$. The lungs were then dried in $80^{\circ} \mathrm{C}$ oven until the weight stayed constant, and the lungs were weighed again to obtain dry weight $(D)$. The W/D ratio was then calculated.

2.9. TNF- $\alpha$ and IL-1 $\beta$ Measurement. The left lung tissues of rats were taken, and bronchoalveolar lavage (BAL) samples were obtained by lavaging the lungs for $1 \mathrm{~min}$ for 3 cycles. The lavage fluid was pooled and kept at $-20^{\circ} \mathrm{C}$ for further use. Enzyme-linked immunosorbent assay (ELISA) was performed according to the instructions of TNF- $\alpha$ and IL- $1 \beta$ ELISA kits. The absorbance was measured at $450 \mathrm{~nm}$, and the concentration of proteins was calculated based on their standard curves.

2.10. Western Bolt. The lung tissues of rats in each group were collected, excised, and washed three times with precooled PBS. The tissues were lysed and homogenized. The lysate of tissue was centrifuged, and the top clear supernatant was electrophoresed by SDS-PAGE. The protein strip was then transferred to the PVDF membrane and blocked with 5\% skim milk. After blocking, the membrane was washed 5 times for 5 min each using $1 \mathrm{X}$ TBST buffer. MCP-1 antibody (1:1000 dilution) and $\beta$-actin antibody (1:1000 dilution) were added to the membrane and incubated at $4^{\circ} \mathrm{C}$ overnight. After washing 5 times with TBST, a secondary antibody was added at a concentration of $1: 5000$ and incubated for 1 hour at $37^{\circ} \mathrm{C}$. ECL developer was applied, and the membrane that was subjected to Quantity One Software was used for data analysis.

2.11. RT-PCR. The mRNA expression of MCP-1 was measured by RT-PCR, and $\beta$-actin was included as internal reference. Lung tissues were lysed in Trizol reagent, and then the mixtures were homogenized to ensure complete breakdown of the tissues. The tissue lysates were set still at room temperature for $5 \mathrm{~min}$. Following that, chloroform was added, and the whole mixture was vortexed for $1 \mathrm{~min}$. The mixture was incubated for $3 \mathrm{~min}$ at room temperature and then centrifuged at $12000 \mathrm{~g}$ for $1 \mathrm{~min}$ at $4^{\circ} \mathrm{C}$. The top clear layer was carefully transferred into a new RNAse free Eppendorf tube, and an equal amount of isopropanol was added to precipitate RNA. The samples were centrifuged for $15 \mathrm{~min}$ to participate the RNA pellets. Each pellet was washed once with $75 \%$ ethanol, air dried, and then dissolved in RNAse-free water. The concentration and purity of RNA

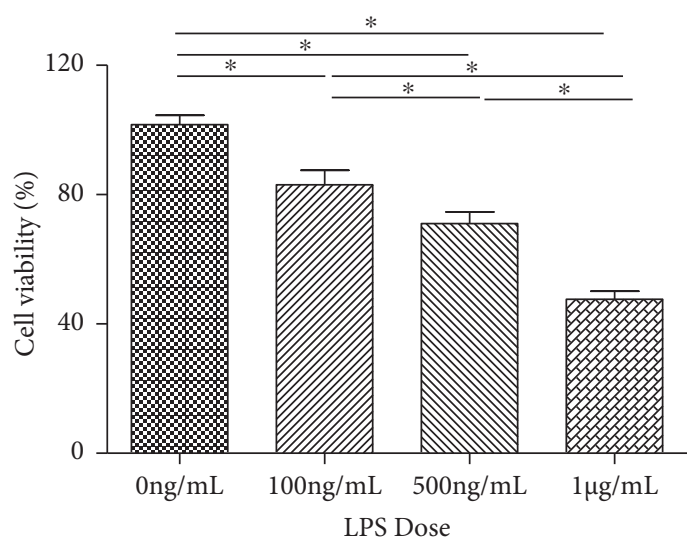

FIGURE 1: Effects of LPS doses on human pulmonary artery endothelial cell viability.

were determined by using Nanodrop. PCR was performed to amplify the cDNA of the target gene. RT-PCR was used to determine the expression of MCP-1 mRNA in each group.

2.12. Statistical Analysis. SPSS 18 software was used for statistical analysis. Data were presented in mean \pm standard deviation format. Between-group comparisons were carried out by one-way analysis of variance (One-way ANOVA). Multiple-group comparisons were conducted by $F$ test. A significant level of each test was set to be $P<0.05$.

\section{Results}

3.1. Characterization of NPs. In this study, the LE of nanoparticle was $23.1 \pm 7.1 \%$. The EE of RS102895 nanoparticle was $40.4 \pm 4.7 \%$. The average size of RS102895 nanoparticle was found to be around $145 \pm 32.9 \mathrm{~nm}$.

3.2. Cell Viability. Compared with the control group (0 ng/mL LPS), the HPAEC cell viability significantly decreased in response to the increased dose of LPS interventions. The dosage of LPS interventions was $100 \mathrm{ng} / \mathrm{mL}$, $500 \mathrm{ng} / \mathrm{ml}$, and $1 \mu \mathrm{g} / \mathrm{mL}$, correspondingly, the cell viabilities were $83 \pm 4.58,71.00 \pm 3.61$, and $47.67 \pm 2.51$. The differences among each group were significant $(P<0.05)$ (Figure 1).

3.3. Cell Apoptosis. To further confirm the effect of LPS on cell viability, Annexin V-APC/7-AAD flow cytometry was used to detect cell apoptosis. The percentages of apoptosis were $37.10 \pm 1.73$ and $46.27 \pm 1.55$ after $100 \mathrm{ng} / \mathrm{ml}$ and $500 \mathrm{ng} / \mathrm{ml}$ LPS interventions (Figure 2). The difference between the two groups was statistically significant $(P<0.05)$. With a further increase of LPS dose, the apoptosis percentage increased to $54.60 \pm 2.80$, which is statistically higher than those of $100 \mathrm{ng} / \mathrm{mL}$ and $500 \mathrm{ng} / \mathrm{mL}(P<0.05)$.

3.4. Blood Gas Analysis. According to the blood gas analysis data, $\mathrm{PaO}_{2}$ and $\mathrm{PaO}_{2} / \mathrm{FiO}_{2}$ of LPS group were lower than those of NC group; the difference between the groups was statistically significant $(P<0.05)$. The $\mathrm{PaO}_{2} / \mathrm{FiO}_{2}$ of LPS group was less than $300 \mathrm{mmHg}$, which met the clinical diagnostic standard of ALI. $\mathrm{PaO}_{2}$ and $\mathrm{PaO}_{2} / \mathrm{FiO}_{2}$ of group $\mathrm{R}+\mathrm{L}$ 


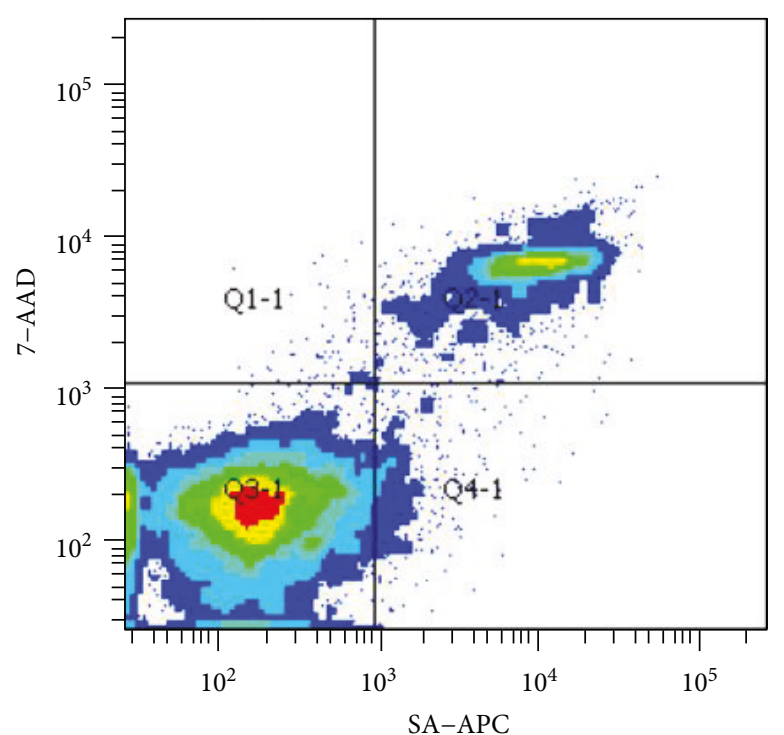

(a)

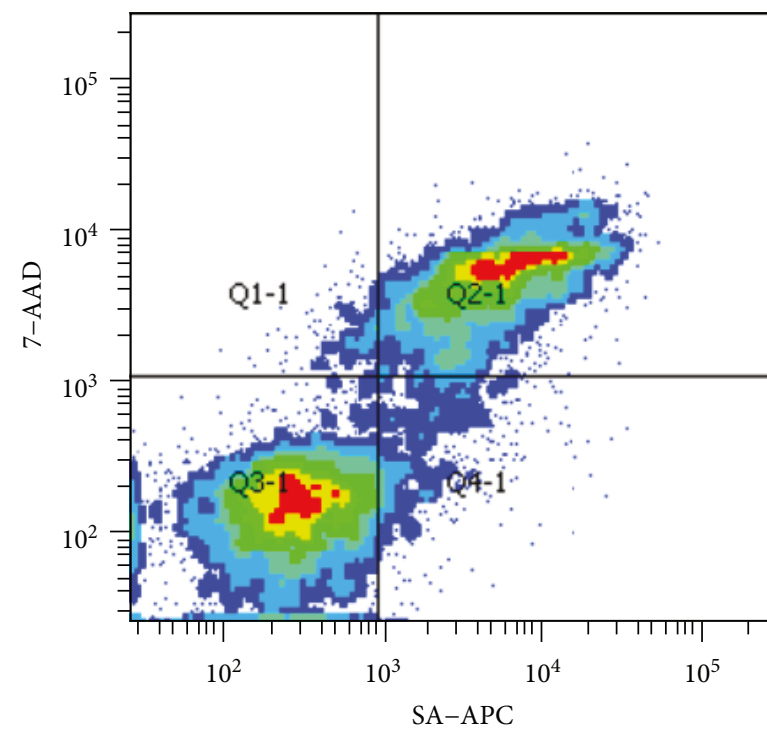

(c)

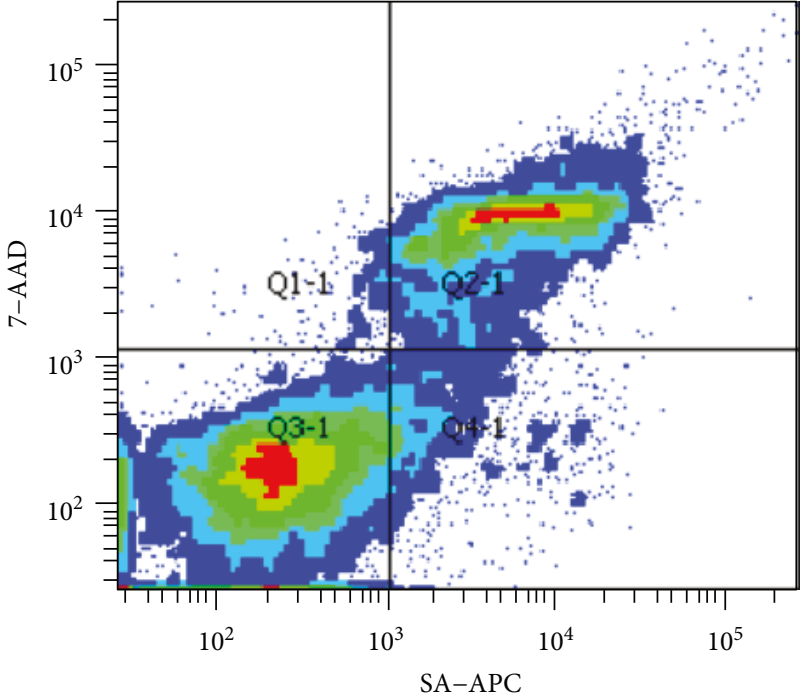

(b)

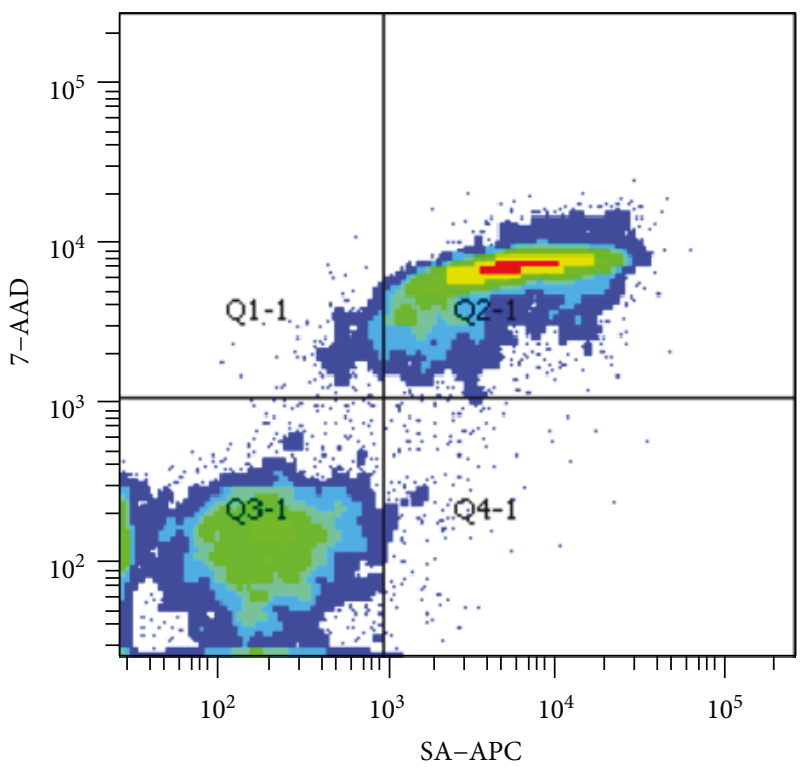

(d)

FIgURE 2: Effects of LPS doses on human pulmonary artery endothelial cell apoptosis. (a) Negative control (LPS $0 \mathrm{ng} / \mathrm{mL}$ ); the rate of apoptosis of cells without LPS stimulating. (b) The rate of apoptosis of cells with LPS stimulating in $100 \mathrm{ng} / \mathrm{mL}$. (c) The rate of apoptosis of cells with LPS stimulating in $500 \mathrm{ng} / \mathrm{mL}$. (d) The rate of apoptosis of cells with LPS stimulating in $1 \mu \mathrm{g} / \mathrm{mL}$.

were significantly higher than group L but significantly lower than those of NC group $(P<0.05)$ (Table 1). Compared to group $\mathrm{R}+\mathrm{L}, \mathrm{PaO}_{2}$ and $\mathrm{PaO}_{2} / \mathrm{FiO}_{2}$ of group $\mathrm{DNPR}+\mathrm{L}$ were significantly lower $(P<0.05)$ (Table 1$)$.

3.5. Lung Wet/Dry Weight Ratio. 24 hours after LPS induced in ALI models, rats in group L were in poor physiological conditions with low activity and poor mental state, while the activities of rats in the NC group were normal. The lung wet/dry weight ratio of LPS group was significantly higher than that of the NC group $(P<0.05)$. The lung wet/dry weight ratio of group $\mathrm{R}+\mathrm{L}$ was significantly lower than group L but significantly higher than the NC group $(P<0.05)$. The lung wet/dry weight ratio of group
DNPR $+\mathrm{L}$ was significantly decreased compared to group $\mathrm{R}+\mathrm{L}(P<0.05)($ Table 1$)$.

3.6. TNF- $\alpha$ and IL-1 $\beta$ Measurement. The level of TNF- $\alpha$ and IL- $1 \beta$ in BALF were measured by using commercial ELISA kits. The concentrations of TNF- $\alpha$ and IL- $1 \beta$ in BALF significantly increased in the LPS group compared with the NC group $(P<0.05)$. The concentration of TNF- $\alpha$ increased from $61.51 \pm 6.22$ to $440.34 \pm 29.06$, while the concentration of IL- $1 \beta$ increased from $45.38 \pm 4.55$ to $341.19 \pm 27.51$. Antagonist RS102895 pretreatment remarkably decreased the levels of TNF- $\alpha$ and IL- $1 \beta$ compared to LPS alone group $(P<0.05)$ (Table 2). Concentration levels of TNF- $\alpha$ and 
TABLE 1: Effects of LPS and LPS/CCR2 antagonist interventions on $\mathrm{PaO}_{2}, \mathrm{PaO}_{2} / \mathrm{FiO}_{2}$, and wet/dry weight ratio in LPS-induce ALI rat models $(\bar{x} \pm s, n=10)$.

\begin{tabular}{lccc}
\hline Group & $\begin{array}{c}\mathrm{PaO}_{2} \\
(\mathrm{mmHg})\end{array}$ & $\begin{array}{c}\mathrm{PaO}_{2} / \mathrm{FiO}_{2} \\
(\mathrm{mmHg})\end{array}$ & $\begin{array}{c}\text { Wet/dry } \\
\text { weight ratio }\end{array}$ \\
\hline NC group & $104.30 \pm 7.36$ & $458.70 \pm 12.05$ & $4.05 \pm 0.15$ \\
L group & $61.50 \pm 7.18^{* \#}$ & $261.30 \pm 7.07^{* \#}$ & $6.02 \pm 0.44^{* \#}$ \\
$\mathrm{R}+\mathrm{L}$ group & $83.30 \pm 3.83^{*}$ & $331.30 \pm 9.87^{*}$ & $5.02 \pm 0.33^{*}$ \\
DNPR + L group & $95.78 \pm 4.51^{\#}$ & $379.85 \pm 8.19^{\#}$ & $4.47 \pm 0.43^{\#}$ \\
$F$ value & 114.07 & 1027.386 & 90.662 \\
$P$ value & 0.000 & 0.000 & 0.000 \\
\hline
\end{tabular}

${ }^{*}$ Compared with NC group $(P<0.05)$; \#: compared with $\mathrm{R}+\mathrm{L}$ group $(P<0.05)$

TABLE 2: Effects of LPS and LPS/CCR2 antagonist interventions on TNF- $\alpha$ and IL- $1 \beta$ in LPS-induce ALI rat models by ELISA $(\bar{x} \pm \mathrm{s}$, $n=10)$.

\begin{tabular}{lcc}
\hline Group & TNF- $\alpha(\mathrm{pg} / \mathrm{mL})$ & IL- $1 \beta(\mathrm{pg} / \mathrm{mL})$ \\
\hline NC Group & $61.51 \pm 6.22$ & $45.38 \pm 4.55$ \\
L Group & $440.34 \pm 29.06^{* \#}$ & $341.19 \pm 27.51^{* \#}$ \\
R + L Group & $150.24 \pm 25.55^{*}$ & $101.08 \pm 10.61^{*}$ \\
DNPR + L Group & $120 \pm 21.43^{\#}$ & $86 \pm 9.47^{\#}$ \\
$F$ value & 766.85 & 833.09 \\
$P$ value & 0.000 & 0.000 \\
\hline
\end{tabular}

*Compared with NC group $(P<0.05)$; \#: compared with $\mathrm{R}+\mathrm{L}$ group $(P<0.05)$

IL- $1 \beta$ in group $\mathrm{DNPR}+\mathrm{L}$ were significantly lower than those in group $\mathrm{R}+\mathrm{L}(P<0.05)$.

3.7. The Expression Level of MCP-1 in Lung Tissue. The expression level of MCP-1 significantly increased in the LPS group compared to the control group $(P<0.05)$. The increase was markedly inhibited by the preinjection of RS102895 $(P<0.05)$. However, the inhibition was not effective enough to counteract the MCP-1 increase induced by LPS posttreatment, as the MCP-1 level was still significantly higher than the control group $(P<0.05)$. The expression of the MCP-1 protein in group DNPR $+\mathrm{L}$ was significantly lower than those in group $\mathrm{R}+\mathrm{L}$, but the expression of the MCP-1 protein in group DNPR $+\mathrm{L}$ was still significantly higher than that in NC $(P<0.05, P<0.05$, Figure 3$)$.

3.8. MCP-1 mRNA Expression. The expression of MCP-1 mRNA in lung tissue was examined by real-time fluorescence quantitative PCR. Compared with the NC group, MCP-1 mRNA expression in LPS group increased by $9.13 \pm 1.15$ times, and the difference was statistically significant $(P<0.05)$. Compared with the LPS group, the expression of MCP-1 mRNA in $\mathrm{R}+\mathrm{L}$ group decreased by $2.55 \pm$ 0.43 times, and the difference was statistically significant $(P<0.05)$. In addition, the expression of MCP-1 mRNA in $\mathrm{R}+\mathrm{L}$ group was higher than that in the control group, and the difference was statistically significant $(P<0.05)$.

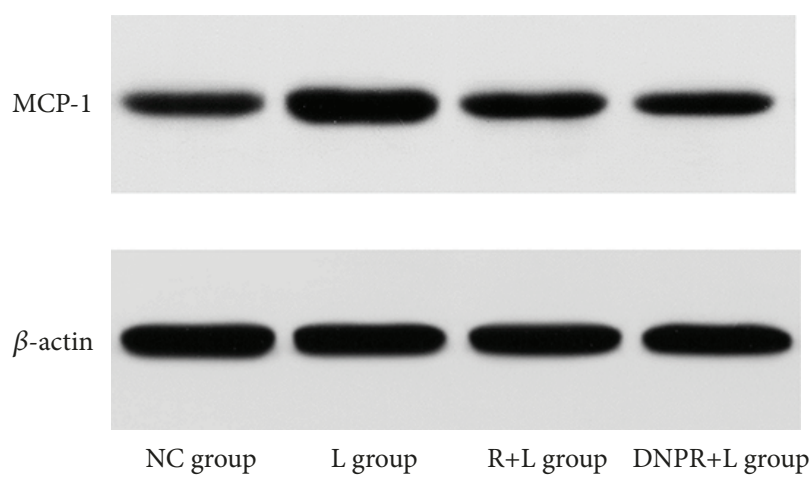

FIGURE 3: Effects of LPS and LPS/CCR2 antagonist interventions on the expression level of MCP-1 by Western blot. The result of Western blot shows that under LPS stimulating the expression is upregulated, and direct administration of RS102895 can reduce the expression of MCP-1 to some extent but not to the condition without LPS stimulating. Nanoparticles can significantly reduce the MCP-1 expression.

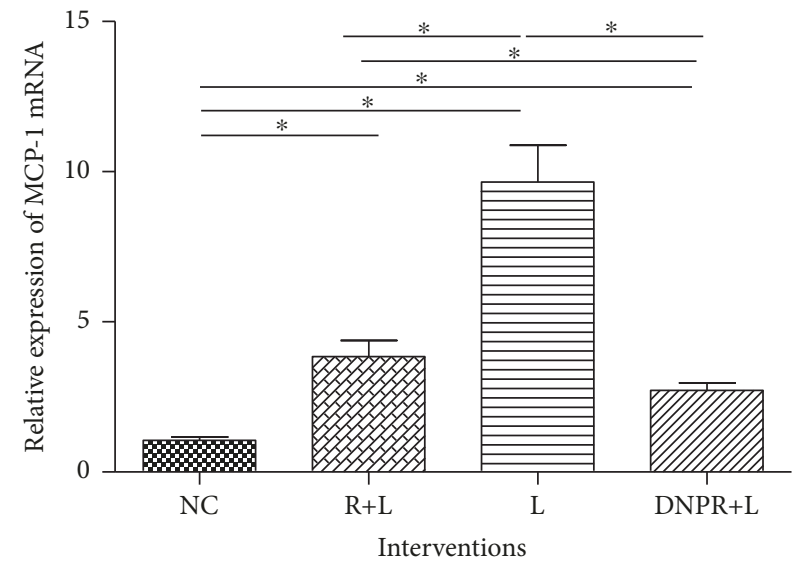

FIGURE 4: Effects of LPS and LPS/CCR2 antagonist interventions on MCP-1 mRNA expression by quantitative real-time PCR.

Compared to NC, the expression of MCP-1 mRNA in group $\mathrm{DNPR}+\mathrm{L}$ significantly increased $(P<0.05$, Figure 4$)$.

\section{Discussion}

ALI is a disease characterized by acute pneumonia and tissue injury [15]. The incidence of the disease is high and the overall mortality rate amounts to $40 \%$ [16]. Bacterial infection is the most common cause of ALI, which may cause lung or systemic inflammation. LPS is the main component of gram-negative bacteria. It is reported that LPS can activate cytokines through a series of cell signal transduction, which promote the release of a large number of inflammatory factors, subsequently induce cell apoptosis, and lead to the occurrence of ALI [17-20]. In this study, in vitro experiments investigated the effects of different doses of LPS $(100 \mathrm{ng} / \mathrm{mL}$, $500 \mathrm{ng} / \mathrm{mL}$, and $1 \mu \mathrm{g} / \mathrm{mL}$ ) on human pulmonary artery endothelial cells. The experimental results showed that LPS can induce an inflammatory reaction. As the LPS dosage 
increased, the cell survival rate decreased gradually along with the apoptosis rate significantly increased $(P<0.05)$.

MCP-1, which belongs to the chemokine family, is a secretory protein that plays an important role in the development of inflammation [21]. Moderate or controlled inflammation is a positive and protective response to multiple injuries, while over or uncontrolled inflammation is harmful and pernicious. Studies have shown that the expression of MCP-1 and MCP-1 mRNA increase significantly when the body suffers chronic and acute inflammations [22, 23]. In addition, the overexpression of MCP-1 can aggravate the occurrence of tissue damage [24]. By binding to its major receptor-CCR2, MCP-1 recruits mononuclear cells, macrophages, and induces cytokine expression [25-27]. MCP-1/ CCR2 pathway is reported to be involved in the pathogenesis of cardiovascular diseases, diabetes, transplantation, and cancer [28-30]. Kalnins et al. suggested that interruption of MCP-1/CCR2 interaction is a promising immunosuppressive therapy for heart transplantation in rats [31]. Gibon et al. also proposed that inhibition of the interaction between MCP-1 and CCR2 weakened their cell chemotaxis abilities [32]. Here, our in vivo study also provided evidence that the blockage of this ligand-receptor axis by CCR antagonists not only effectively reduces the levels of inflammatory cytokines but also reduces chemokines, which, overall, attenuates acute lung injury symptoms in LPS-induced ALI rat model. However, during our study, we found out that direct administration of the antagonist RS102895 is not effective enough for treatment. Therefore, we alternated the way for delivering the inhibitor. We polymerize dextran into polyaldehyde dextran and taken this polymer as a vector vehicle to transfer the inhibitor into intracellular space, and with the help of the nanoparticles, the inhibit efficiency raised from $60 \%$ to $80 \%$.

However, our results also indicated that the protective effects of MCP-1/CCR2 blockage cannot counteract the effects of LPS challenge evidenced by significant differences between $\mathrm{R}+\mathrm{L}$ group and $\mathrm{NC}$ group. The MCP-1/CCR2 pathway is only one of many pathways in systematic disease such as acute lung injury. LPS is recognized by toll-like receptors on the membrane of antigen-presenting cells, which triggers LPS/TLR/MyD88/IRAK/TRAF/NF- $\kappa$ B signaling pathway $[33,34]$. The activation of NF- $\kappa \mathrm{B}$ results in more inflammatory cytokines and chemokines such as TNF- $\alpha$, IL-1 $\beta$, and MCP-1. If the MCP-1 expression level keeps increasing, more leukocytes would be attracted accompanied by an increase of TNF- $\alpha$ and IL- $1 \beta$. This loop is endless and overwhelming. Interventions which are able to inhibit LPS/TLR/MyD88/NF- $\kappa \mathrm{B}$ signaling pathway must be more effective to prevent the negative consequences.

In conclusion, the MCP-1/CCR2 signaling contributes to the pathogenesis of acute lung injury. In order to test whether MCP-1/CCR2 pathway could be a potential therapeutic target for ALI treatment and molecules interfering with MCP-1/CCR2 interaction could be studied and regarded as a promising ALI therapy, future clinical trials should be taken into consideration. And our method to deliver the inhibitor through self-synthesized nanoparticles may provide a more efficient way for drug delivery. Furthermore, aside from inhibition of MCP-1/CCR2 interaction, inhibition of TLR/
MyD88/NF- $\kappa \mathrm{B}$ signaling pathway might be a more promising treatment for acute lung injury.

\section{Data Availability}

The data used to support the findings of this study are available from the corresponding author upon request.

\section{Ethical Approval}

Approval for the present study was obtained by the Ethics Committee of Shanghai Ninth People's Hospital (Shanghai, China).

\section{Consent}

All subjects participating in the image acquisition signed the consent form.

\section{Conflicts of Interest}

We declare that we have no financial and personal relationships with other people or organizations.

\section{Authors' Contributions}

Zheng Cao designed the study and performed the experiments. Qiao Wang analyzed the data. All authors read and approved the manuscript. Qiao Wang was responsible for study conception and design and revised the manuscript; Zheng Cao and Jing-Lan Liu performed the experiments and drafted the manuscript; and Zheng Cao and Shen $\mathrm{Wu}$ analyzed the data. All authors read and approved the final manuscript.

\section{Acknowledgments}

The article is supported by grant 51673190 from the National Natural Science Foundation of China, and the data and materials used to support the findings of this study are included in the published article.

\section{References}

[1] G. D. Rubenfeld, E. Caldwell, E. Peabody et al., "Incidence and outcomes of acute lung injury," The New England Journal of Medicine, vol. 353, no. 16, pp. 1685-1693, 2005.

[2] N. B. Yu, "Therapeutic experience of the application of anisodamine on acute lung injury," Journal of Acute Disease, vol. 5, no. 5, pp. 382-385, 2016.

[3] G. M. Mutlu and G. R. Budinger, "Incidence and outcomes of acute lung injury," The New England Journal of Medicine, vol. 354, no. 4, pp. 416-417, 2006.

[4] P. Toy, O. Gajic, P. Bacchetti et al., "Transfusion-related acute lung injury: incidence and risk factors," Blood, vol. 119, no. 7, pp. 1757-1767, 2012.

[5] F. Dias-Freitas, C. Metelo-Coimbra, and R. RonconAlbuquerque Jr., "Molecular mechanisms underlying hyperoxia acute lung injury," Respiratory Medicine, vol. 119, pp. 23-28, 2016. 
[6] V. von Dossow-Hanfstingl, "Advances in therapy for acute lung injury," Anesthesiology Clinics, vol. 30, no. 4, pp. 629639, 2012.

[7] S. Guo, Y. Fu, S. Xiong, and J. Lv, "Corilagin protects the acute lung injury by ameliorating the apoptosis pathway," Biomedicine \& Pharmacotherapy, vol. 95, pp. 1743-1748, 2017.

[8] S. Rajasekaran, D. Pattarayan, P. Rajaguru, P. S. Sudhakar Gandhi, and R. K. Thimmulappa, "MicroRNA regulation of acute lung injury and acute respiratory distress syndrome," Journal of Cellular Physiology, vol. 231, no. 10, pp. 2097-2106, 2016.

[9] S. Herold, K. Mayer, and J. Lohmeyer, "Acute lung injury: how macrophages orchestrate resolution of inflammation and tissue repair," Frontiers in Immunology, vol. 2, p. 65, 2011.

[10] C. H. Han, P. X. Zhang, and W. W. Liu, "Macrophage polarization is related to the pathogenesis of decompression induced lung injury," Medical Gas Research, vol. 7, no. 3, pp. 220-223, 2017.

[11] P. Störmann, T. Lustenberger, B. Relja, I. Marzi, and S. Wutzler, "Role of biomarkers in acute traumatic lung injury,” Injury, vol. 48, no. 11, pp. 2400-2406, 2017.

[12] M. Yang, J. Ding, X. Feng et al., "Scavenger receptor-mediated targeted treatment of collagen-induced arthritis by dextran sulfate-methotrexate prodrug," Theranostics, vol. 7, no. 1, pp. 97-105, 2017.

[13] Y. Zhang, F. Wang, M. Li et al., "Self-stabilized hyaluronate nanogel for intracellular codelivery of doxorubicin and cisplatin to osteosarcoma," Advanced Science, vol. 5, no. 5, article 1700821, 2018.

[14] N. Lomis, S. Westfall, L. Farahdel, M. Malhotra, D. Shum-Tim, and S. Prakash, "Human serum albumin nanoparticles for use in cancer drug delivery: process optimization and in vitro characterization," Nanomaterials, vol. 6, no. 6, p. 116, 2016.

[15] A. G. Proudfoot, D. F. McAuley, M. J. D. Griffiths, and M. Hind, "Human models of acute lung injury," Disease Models \& Mechanisms, vol. 4, no. 2, pp. 145-153, 2011.

[16] Y. Cao, Y. Lyu, J. Tang, and Y. Li, "MicroRNAs: novel regulatory molecules in acute lung injury/acute respiratory distress syndrome," Biomedical Reports, vol. 4, no. 5, pp. 523527, 2016.

[17] J. Lei, Y. Wei, P. Song et al., "Cordycepin inhibits LPS-induced acute lung injury by inhibiting inflammation and oxidative stress," European Journal of Pharmacology, vol. 818, pp. 110$114,2018$.

[18] N. A. Maniatis, A. Kotanidou, J. D. Catravas, and S. E. Orfanos, "Endothelial pathomechanisms in acute lung injury," Vascular Pharmacology, vol. 49, no. 4-6, pp. 119-133, 2008.

[19] Y. L. Wang, X. Y. Guo, W. He, R. J. Chen, and R. Zhuang, "Effects of alliin on LPS-induced acute lung injury by activating PPAR $\gamma$," Microbial Pathogenesis, vol. 110, pp. 375$379,2017$.

[20] G. Deng, H. He, Z. Chen et al., "Lianqinjiedu decoction attenuates LPS-induced inflammation and acute lung injury in rats via TLR4/NF- $\kappa$ B pathway," Biomedicine \& Pharmacotherapy, vol. 96, pp. 148-152, 2017.

[21] A. Yadav, V. Saini, and S. Arora, "MCP-1: chemoattractant with a role beyond immunity: a review," Clinica Chimica Acta, vol. 411, no. 21-22, pp. 1570-1579, 2010.

[22] J. Panee, "Monocyte chemoattractant protein 1 (MCP-1) in obesity and diabetes," Cytokine, vol. 60, no. 1, pp. 1-12, 2012.
[23] J. N. Hilda and S. D. Das, "TLR stimulation of human neutrophils lead to increased release of MCP-1, MIP- $1 \alpha$, IL- $1 \beta$, IL- 8 and TNF during tuberculosis," Human Immunology, vol. 77, no. 1, pp. 63-67, 2016.

[24] E. A. Boström, E. Kindstedt, R. Sulniute et al., "Increased eotaxin and MCP-1 levels in serum from individuals with periodontitis and in human gingival fibroblasts exposed to pro-inflammatory cytokines," PLoS One, vol. 10, no. 8, article e0134608, 2015.

[25] Y. Hu, N. D. Kodithuwakku, L. Zhou et al., "Levo-corydalmine alleviates neuropathic cancer pain induced by tumor compression via the CCL2/CCR2 pathway," Molecules, vol. 22, no. $6,2017$.

[26] H. Zhang, Y. Li, M. de Carvalho-Barbosa et al., "Dorsal root ganglion infiltration by macrophages contributes to paclitaxel chemotherapy-induced peripheral neuropathy," The Journal of Pain, vol. 17, no. 7, pp. 775-786, 2016.

[27] S. Al-Mazidi, M. Alotaibi, T. Nedjadi, A. Chaudhary, M. Alzoghaibi, and L. Djouhri, "Blocking of cytokines signalling attenuates evoked and spontaneous neuropathic pain behaviours in the paclitaxel rat model of chemotherapyinduced neuropathy," European Journal of Pain, vol. 22, no. 4, pp. 810-821, 2018.

[28] J. Kalliomäki, B. Jonzon, K. Huizar, M. O’Malley, A. Andersson, and D. M. Simpson, "Evaluation of a novel chemokine receptor 2 (CCR2)-antagonist in painful diabetic polyneuropathy," Scandinavian Journal of Pain, vol. 4, no. 2, pp. 77-83, 2013.

[29] Y. He, X. Xu, T. Zhu, M. Tang, J. Mei, and Y. Si, "Resident arterial cells and circulating bone marrow-derived cells both contribute to intimal hyperplasia in a rat allograft carotid transplantation model," Cellular Physiology and Biochemistry, vol. 42, no. 4, pp. 1303-1312, 2017.

[30] Y. Wang, H. Ni, H. Li et al., "Nuclear factor kappa B regulated monocyte chemoattractant protein-1/chemokine CC motif receptor-2 expressing in spinal cord contributes to the maintenance of cancer-induced bone pain in rats," Molecular Pain, vol. 14, 2018.

[31] A. Kalnins, M. N. Thomas, M. Andrassy et al., "Spiegelmer inhibition of MCP-1/CCR2 - potential as an adjunct immunosuppressive therapy in transplantation," Scandinavian Journal of Immunology, vol. 82, no. 2, pp. 102-109, 2015.

[32] E. Gibon, T. Ma, P. G. Ren et al., "Selective inhibition of the MCP-1-CCR2 ligand-receptor axis decreases systemic trafficking of macrophages in the presence of UHMWPE particles," Journal of Orthopaedic Research, vol. 30, no. 4, pp. 547-553, 2012.

[33] Y. Luo, W. Che, and M. Zhao, "Ulinastatin post-treatment attenuates lipopolysaccharide-induced acute lung injury in rats and human alveolar epithelial cells," International Journal of Molecular Medicine, vol. 39, no. 2, pp. 297-306, 2017.

[34] X. Ding, S. Jin, Y. Tong et al., “TLR4 signaling induces TLR3 up-regulation in alveolar macrophages during acute lung injury," Scientific Reports, vol. 7, no. 1, article 34278, 2017. 


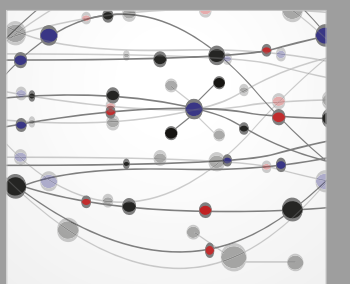

The Scientific World Journal
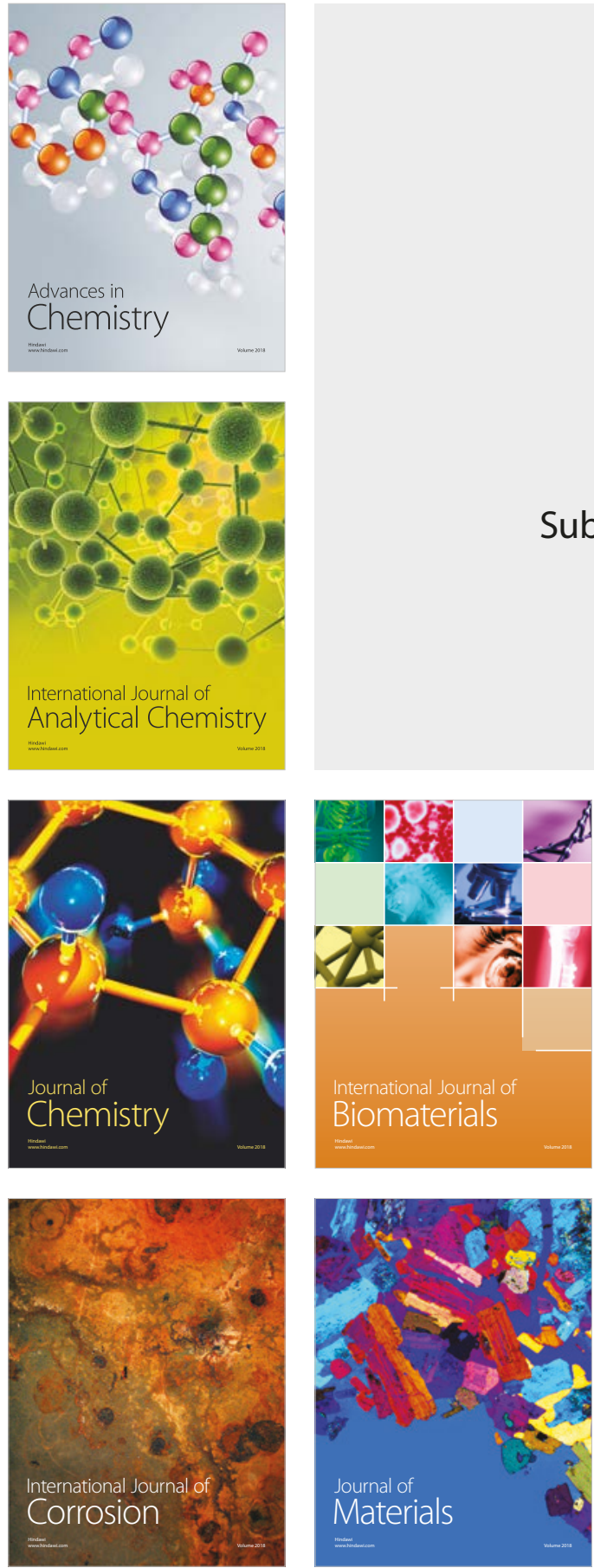

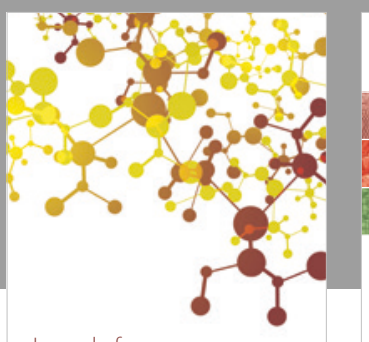

Journal of

Applied Chemistry
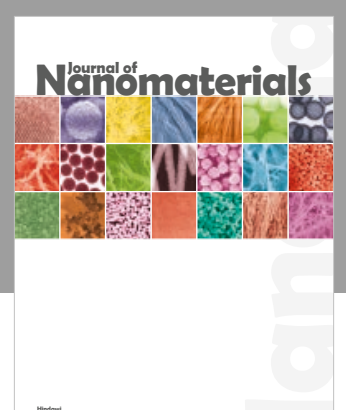

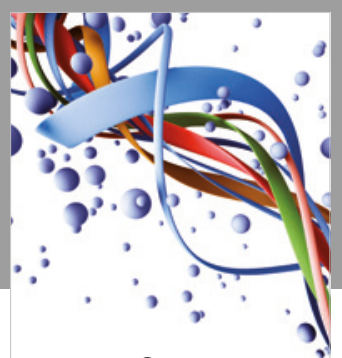

Scientifica

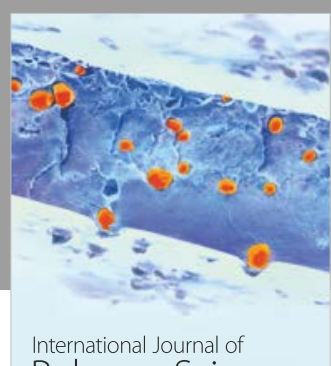

Polymer Science

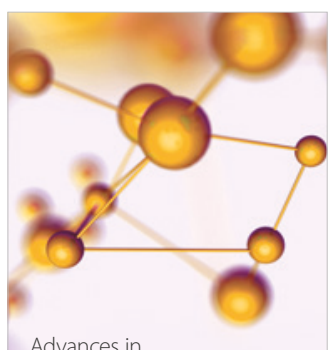

Physical Chemistry
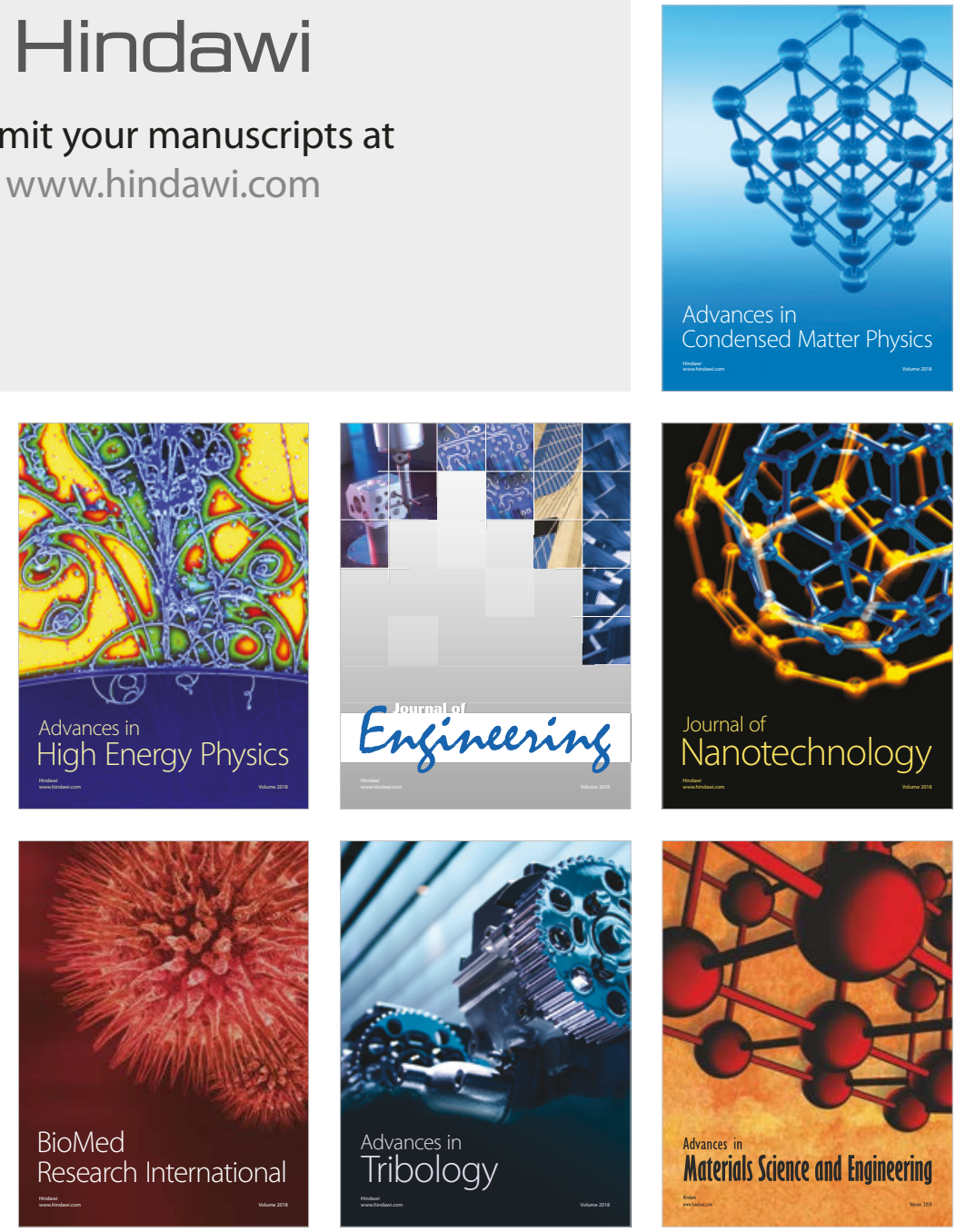